\title{
Geo Location based Task Management with Path Optimization
}

\author{
A.Vincy \\ Masters Student \\ Apollo Engineering College \\ Chennai, India
}

\author{
S. Prasanna Devi \\ Head of Department \\ Apollo Engineering College \\ Chennai, India
}

\begin{abstract}
Objective of this paper is to build an application using which tasks can be managed based on geographic location in an optimized manner. The project uses geo location mapping using GPS enabled smart phones, integrated map API and path optimization. In today's world smart mobile phone devices has become an integral part of people life. Among its various uses, building application to support location based services is a trend now a days. Using this LBS concept, this paper deals with the process of task management. Scheduling an appointment or a task and configure it to remind on a scheduled time has become an obvious regular activity. Even though date and time is the core parameters of task management, there is a gap in it. Certain tasks cannot be scheduled based on pre-determined time but can be associated with a location. So, the reminder systems were evolved by considering locations instead of date and time. But, all of them have eminent problems. So the aim of this paper is to enhance task management using location based service using latest technologies and to solve the problem of existing system and also to enable users to manage their daily tasks with better planning. Added to that, this paper is about to cover maximum number of tasks with minimum distances with a representation of map which will be shown to the user along with the alarm when they cross their location of interest.
\end{abstract}

\section{General Terms}

Geo Spatial Services.

\section{Keywords}

m-learing, map mashups, Location Reminder, Context Aware Services, GPS

\section{INTRODUCTION}

Location based service is the one which compute location with the help of the location providers. There are two widely used location providers named GPS provider and network location provider. The GPS provider picks location by extending GIS capabilities and it needs no internet connection whereas the network location provider picks location based on the cell phone tower. So it needs any one of the mobile networks or WiFi to be enabled. The location details are taken as the two parameters such as latitude and longitude. These parameters will be converted in the form of address or anything depends on the requirement of the service created.

These LBS services and the concept about location learning or $\mathrm{m}$-learning are attracted by the people now days. In short time, these services became much popular, by supporting mobile system users. So, with the compact, portable primary hardware like smart phones, tablet and etc, people can go with such supportive services which help them by reducing their burden. Even though the services for context aware devices are more in number already, the innovative new services with enhancement are appreciated by the user.

Usually location based services are very popular by performing some event required by the user. But generally, these services are provided to target somewhat limited population of specific domain. For example, "Forget-me-not" application is one of the location based services that will provide the ability to remain about the activities yet to be completed in organization level. And "Tour-Guide "and "Mobile Tourism Directory" will provide tour information services by people-centric computing.

Unlike some domain specific location based services, this project is used to provide a product to all users in common. This is supposed to remind people about their daily tasks depend on the location they are crossing on. In today's modern machine world, forgetting our task to be completed that is quite natural. So, this paper deals with an idea which will create revolutionary effect among the existing location based services.

Recently, smart phones are most passionate equipment of most of the user. It plays major role as part of their daily life. So this service is provided as a mobile application. It leads better task planning based on the user's current location. The task management is performed by sending alert to the user with the task details needed to be noticed. On clicking the notification sent, a graphical plan will be shown to the user by covering maximum possible number of task around his current location.

In the following pages, we are going to discuss about the background knowledge of LBS, existing system working under the concept of Location based learning, System architecture of proposed system and its requirement depends on the user, above all the basic functionalities comprised with implementation of the system.

\section{EVOLUTION OF LBS}

Location-based services are the important application component that are used for the operations like location based search, social networking between users where their location will be change periodically. And also supports the computation of the continuous trace upon the navigation with increasing awareness of the point of location of the mobile devises among the geographic plane. Based on these capability, there are many applications are created. For example, some application in the domain of health care management to raise a location based alert based on the patient critical position during his travel. To extend the focus independent of the domain this paper deals with the application to facilitate the users of all domain in common by supporting people to remind their daily task by raising location based remainders. The following pages say about the 
various location based services available for the last few years.

In "Forget-me-not" application, it uses intimate computing model to monitor users activity in different location and to save information from different devices people using. It needs a portable device which acts as a intimate computer. In older days PDAs were used as the intimate computers. Then they were changed by the slight improvement of technology. So the PDAs are changed by the mobile devices for example Cell Phones in recent days. This results the impact of the user of this application by the advanced development in mobile computing. So, it is understandable that the advancement in development should not affect the application for longer run. But the intimate computer needs not to store all the information into its own memory always. Rather it can retrieves activity details on need basis. It helps people to remember about specific things based on location. The interface is created by some wearable small devices like wrist watch. A small display will be there at this device. The program for forget me not and data is reside into this interface. The details are passed as symbols which are not easy to read. And the location information will be retrieved by the room based transceivers. So this application is useful within some restricted boundary. Above all this idea is implemented with more hardware devices and has some complexity.

In [5] the Location-aware information delivery with commotion 2000, maps users task to be completed with location they are on particular time. For example, this will remind the list of tasks at bank when we are there. This will be accomplished by satellite based GPS position sensor. The focus is on location-aware computing environment where the personal information and user's locations are linked together to make people to know their current location. This complete set of response to be sent to the people that will be created by the uses of graphical user interface. However, because it is intended primarily for mobile use, including driving, the core set of reminder creation and retrieval can be managed completely by speech. Evaluation has to be improved over the functionalities, location learning, and information delivery.

In [2] the "Place-Its" application had come with some ideas to implement the LBR services with some feature enhancement of the previous services. This application mainly focuses on remembering future task by raising alarms using location aware mobile applications. It has some limitations over space and location accuracy. This application had capture the point of view about how the ubiquities system creates the effect on person's natural setting with the role of contextual remainders. Mobile phones with location-sensing capabilities are becoming state of the art, for deploying context-aware reminder applications. Because the ubiquity of mobile phone networks enables pervasive location sensing, while the always-carried and always-on nature of phones mean that reminder creation and notification are permanently available to users. But, the sensing capabilities of phones are limited and are not guaranteed based on the context type to be sensed and also based on the level of accuracy. With very less input domain space mapped with another task does not facilitate with the ease of use. The resulting product had been in preliminary stage. Some more improvement is needed with this result based on the limited text entry mechanisms available, a way of associating audio messages or pictures with reminders could offer greater convenience, encouraging unique and more opportunistic use. And also, the effects of inaccurate location sensing and naturally support the use of recurring reminders and on the change to the user interface need to be taken in to account.

In [3] there are some case studies to support location based reminder concept for its implementation. Such studies helped to create the PlaceMail application which was expected to manage everyday tasks. People used to manage distributed tasks and note problems with current methods, including the common "to-do list". PlaceMail supports useful locationbased reminders and functional place-based lists. But this has some issues related when and where to deliver location-based information, and also has insufficient response while creating the traditional 'geofence' radius around a place. Instead, output is depended on people's movement patterns through an area and the geographic layout of the space. Our results both provide a compelling demonstration of the utility of locationbased information and raise significant new challenges for location-based information distribution.

In [4] the CenseMe-application in 2008, enables social network users to share personal information by classifying sensed data. The information like what the person is doing can be shared. For example, if a person is attending a conference and so on. This is for an app which makes comprehensive use of facilities offered by the devices based on the sensed information. Besides the ability to share the current location, it uses algorithms to classify the sensed data and recognizes whether the user is alone, attending a meeting or such information like, driving or running, etc. . The integration of such a service into a task planning application is interesting in terms of group or office management.

All the location based services should show the user's current location or some other location of his interest based on the application context, with some pictorial representation. For that some of the mashup applications were developed. The best example for those things is Open Street Map (OSM) and the revolutionary product Google Map, Google Earth and etc. In [9], "Map Mashups Web 2.0 and the GIS revolution" in the year of 2010 , deals with the idea or with some preliminary works to create blending of several mashup software with the emergence of Web 2.0 and GIS technology. A new type of geography called 'Neogeography' is used to make normal, non-expertise people to get advanced application of map.

\section{EXISTING WORKING SYSTEM}

The existing system is a Geo Temporal Task Planning Application named as LatYourLife [10] and simply called as LYL. It connects people's daily tasks with geographical information stored in their devices. The main focus is to plan people's tasks as per the location they are crossing on. This task planning has been accomplished by the group of components as web interface, spatial database, and mobile application. The spatial database is connected with either web interface or with mobile application based on the devices used by the user.

A peculiar decision making process is needed to take with the services like predicting what is around, routing, finding the nearest path and finally handling events at right time to raise proactive alert. The enhancement over the previous work before LatYourLife was having an idea of computing when to act upon the process of triggering the alerts depends upon the weather results returned by the cloud system called google weather API.

System phases are subdivided into three phases. They are before phase, during phase and after phase. In before phase the decision making process will be done before triggering the 
alarm. In during phase, it is collected that the set of requirements are enough to achieve the goal. And in after phase includes the review process to ensure the measured requirements are enough to achieve the goal.

This application reduces human work on measuring the parameter to create a plan with the map where the users locations and its corresponding tasks to be covered in this area will be instructed. These parameters are what-is-around, routing process, nearest path services, and when-to-act. And also, it has very good feedback from users for web interface which was useful with clear visibility of location.

\section{Drawbacks:}

1. It has some problem on raising alarm in appropriate time to remind events.

2. It has complexity over technical implementation of data sensed by the planning phase or before phase.

3. It is not capable of grouping too many tasks to be completed at a moment.

4. Its response is slow in highly densely populated area.

\section{PROPOSED SYSTEM}

\subsection{Architecture}

Figure-1 shows the proposed system architecture. It includes the following component

\section{Primary Hardware \\ 2. Geo Task Planner Application \\ 3. SQLite Database \\ 4. Integrated Google Map API}

\section{Primary Hardware:}

The primary hardware used here is the GPS enabled smart phone as shown in the architectural diagram. Previously the LBS are provided among a particular network whereas the users should have to be connected with a common centralized device. So the number of hardware was too heavy which created frequent hardware component failure. To reduce this problem, the LBS are provided with the mobile devices latter which will increase the sage convenient and portability.

\section{Geo Task Planner Application:}

The Geo Task Planner Application is built on top of it in Android development environment. This environment is the specified android version of java programming. That means, it includes classes, interfaces only for android. After complete development, this application will be launched in to the required android mobile device as directed by the configuration settings applied with the help of the developers IDE platform tools. During this launch, the application will be stored in to the device as an .apk file which will be managed with the android lifecycle process.

\section{SQLite Database:}

There are various storage options in android development environment. Some of them are named as, internal storage, external storage, SQLite database. Among the various types, the SQLite database is the suitable one to build the database related application with ease of development process. And also, this database is private to the specified application which cannot be access by any other component. So it will provide safety over the application data.

SQLite database is like as a relational database using which the Android API helps to perform the typical database CRUD operations from the application components. The link between application component and the database will be created with the use of SQLiteOpenHelper class. It contains the set of call back functions named onCreate() and onUpgrade() to create or upgrade database while launching at first time.

\section{Google Map API:}

Google map API is the revolutionary launch among the google products. Most of the location based applications are built by using this API to visualize the geospatial information on top of the customized map. It provides the ability to the developer not only to the experts to create their own map.

During implementation this paper, Google Map API is used to create a geographical representation of the user's position mapped with the tasks. This representation will be used for good decision making about what to do next, based on where we are. So it is needed to be integrated to tile the location on top of the map for providing better planning about the tasks needed to be covered by the user.

\section{Other Concepts:}

Apart from the software and hardware components specified in this architectural diagram, there are two more concepts used. These are,

\section{Global Positioning System}

\section{Dijkstra's Path Optimization algorithm}

Global Positioning System:

GPS is satellite navigation system to focus and gather the spatiotemporal data around geo space. To support the location based services the devices are made to utilize this navigation system. Such devices are acting as the GPS receivers. They are about to receive the information send by the number of satellite available.

This information includes the sending time and the satellite location. By receiving this message, the receiver can find the distance of each satellite. Using this information the location of the user or the user's devices which acts as GPS receivers.

This calculation is performed by the set of navigation equations. The parameters needed for this equation is the satellite position as $\mathrm{x}, \mathrm{y}, \mathrm{z}$ coordinates as $[\mathrm{xi}, \mathrm{yi}, \mathrm{zi}, \mathrm{ti}]$, where 'i' represents the satellite.

Dijkstra's Path Optimization algorithm:

Dijkstra's algorithm is used here for finding the shortest possible route to cover maximum number of locations. To implement this algorithm some set of processes is needed to traverse among the plotted location after creating the map. The path created by the Google map API is considered as a graph which is sent to this algorithm as an input. The distance between two locations is called as the edge of the graph and the distance is taken as the cost of the edge. Then a shortest path tree will be created.

The main function of this algorithm is to find the route with minimum cost or distance. Sometimes this algorithm is used to create only the shortest path tree. after that the path with minimum cost will be created by another path finding 
algorithm. Dijkstra's search for path finding from a start to destination is a robot motion planning problem. Let the node at which we are starting is called as the initial node. After initialization some set of steps is needed to be performed.

1. Mark each edge with their distance.

2. Mark all nodes as unvisited where current node is the initial one. Group the unvisited nodes together as a set excluding the initial node.
3. From the current node, all of its unvisited neighbours will be identified with their approximate distances. Update the tentative distance by comparing the current and previous node.

4. Mark node as visited once all of its neighbour nodes are calculated.

5. For our consideration, if the destination node has been marked visited then stop otherwise continue step 3 and 4 .

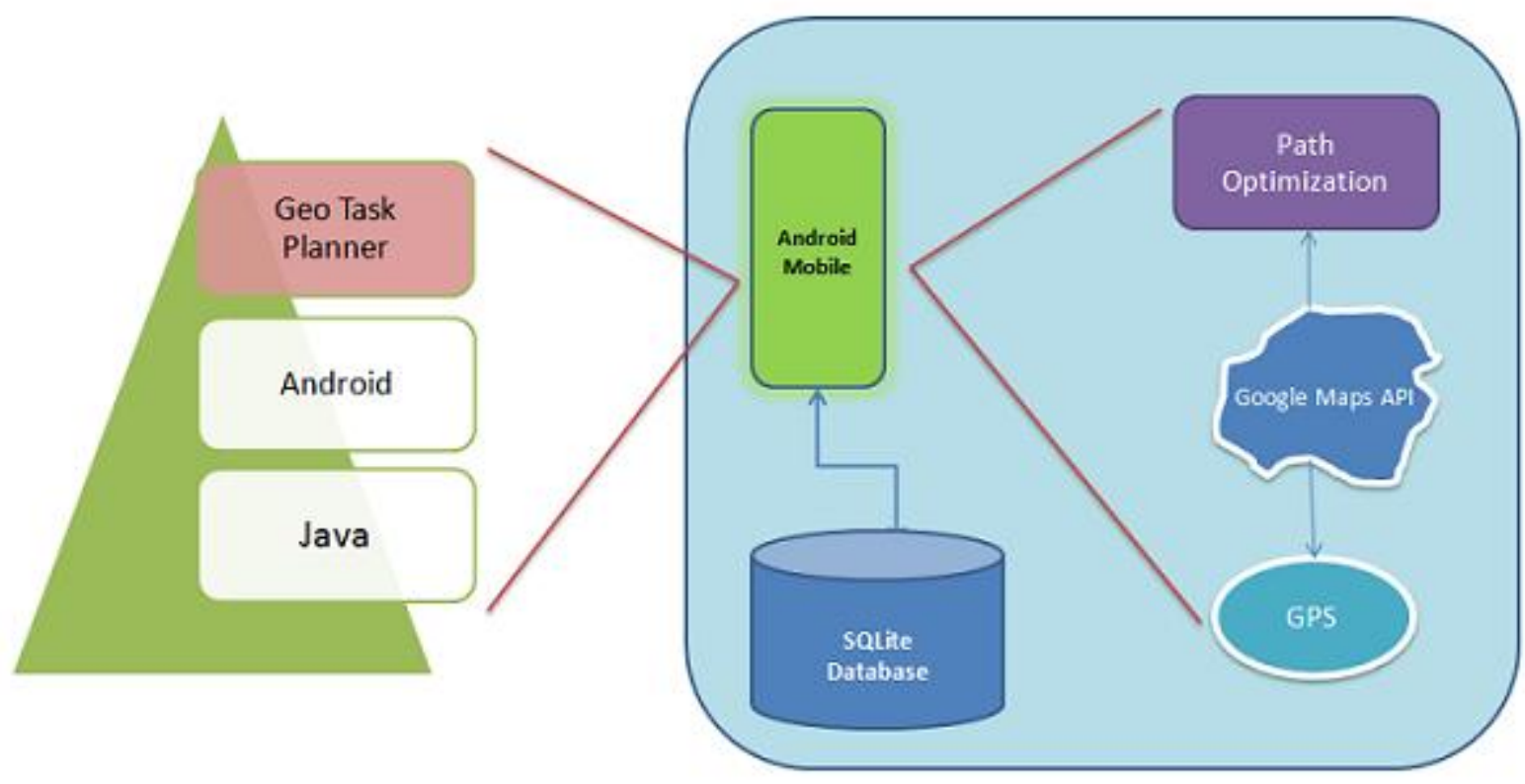

Fig 1: Architecture of Proposed System

\section{SYSTEM REQUIREMENTS}

The main work focus in this paper is to monitor user's location, creating map and optimize the route. So, the system where this idea is to be implemented should have the following requirement.

\section{Android version 2.3 called Gingerbread.}

2.Mobile network and Wi-Fi should be enabled to display the google map.

The above requirements are needed to run the application in real device. If it is needed to run in emulator then the Android Virtual Device should be installed and selected.

\section{FUNCTIONALITIES}

To implement this paper, the following lists of functionalities are essential.
1. Location directory
2. Task Manipulation
3. Map Construction
4. Geo Synchronization

\section{Path Optimization}

6. Generating location based alarm

Among these functionalities, the process of creating location directory and synchronizing the user's current location with the spatio temporal data stored into the database are handled with the help of GIS capabilities. And then, to construct the map it is supported by the google map API. For that, the Map Mashup algorithm [9] is used to create the KML data. This data will be sent as an input to the Google Map API. The output of this functionality will be optimized by applying Dijkstra's shortest path finding algorithm. Then, the remaining functionalities can be covered by the features of Android development environment itself without the needed of any open source integration.

\section{EXPERIMENTAL RESULTS}

After implementing the above functionalities, location of interest based on task stored in database is tiled on to the map. And then, the path is suggested by following street. Following figures shows two possible routes. Figure 2.a shows the nonoptimized route whereas figure $2 . b$ shows the optimized one. 


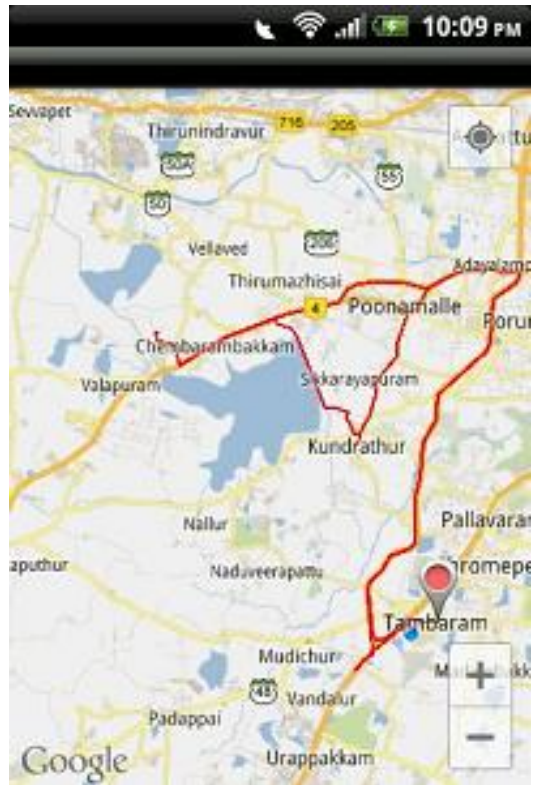

Fig 2.a: Non optimized route

\section{CONCLUSION}

A huge support for human memory is needed essentially to remind the daily tasks to be performed. This project helps people in this regard to gain the maximum utilization of location-based services. Since the reminder sent to the people via their devices is generated based on the time and the location, this project leads better task planning. The reminder may be a flash of light, or any alarm or in some other form based on the user's settings. Since the smart phones are popularly used devices of this generation, this project is developed in the form of mobile application. The Android phones are having greater availability and also having affordable with cost, this project is planned to develop in Android platform to gain greater usability. Among the current location based services available already, this application is enhanced to the level of using the concept named 'Neogeography' to let people to gain the maximum application of map. The main motive to group many task as much as possible on covering less distance. Added to that, it also overcomes the deficiencies of existing application.

\section{REFERENCES}

[1] Lamming M. and Flynn M., "Forget-me-not" Intimate Computing in Support of Human Memory. Proceedings of FRIEND21, 1994 International Symposium.

[2] Sohn T., Li K. A., Lee G., Smith I., Scott J., and Griswold W. G. "Place-Its: A Study of Location-Based Reminders on Mobile Phones" Proc. International Conference of Ubiquitous Computing, 2005.

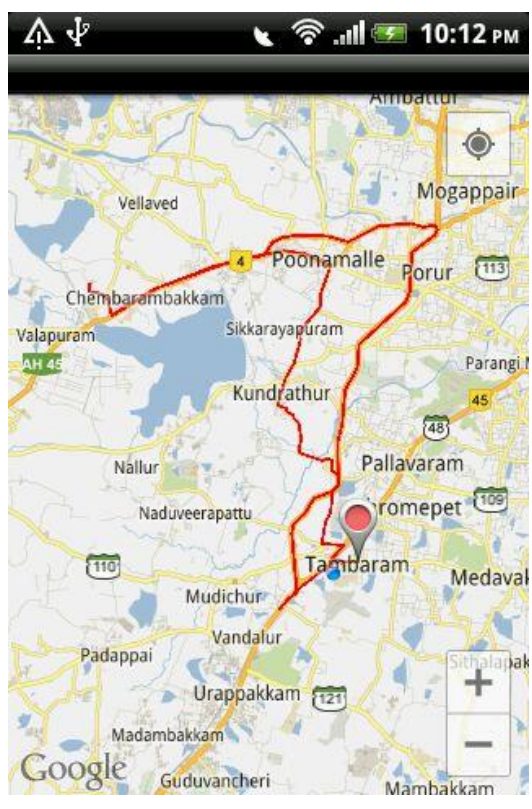

Fig 2.b: Optimized route

[3] Pamela J. Ludford, Dan Frankowski, Ken Reily, Kurt Wilms, Loren Terveen Because I Carry My Cell Phone Anyway: Functional Location-Based Reminder Applications," Proc. ACM SIGCHI, 2006.

[4] Miluzzo E., Lane N. D., Fodor K., Peterson R., Lu H., Musolesi M., Eisenman S. B., Zheng X., and Campbell A. T., "Sensing meets mobile social networks: the design, implementation and evaluation of the CenceMe application," Proceedings of the 6th ACM, 2008.

[5] Marmasse N. and Schmandt C.: "Location-aware information delivery with commotion," Proceedings of the 2nd international symposium on Handheld and Ubiquitous Computing, 2000.

[6] Mobile Location-Based Learning Reminders using GSM cell Identification 2009, IADIS International Journal on WWW/Internet.

[7] Geolearners: Location-Based Informal Learning with Mobile and Social Technologies 2010, IEEE Transactions on Learning Technologies.

[8] Mobile Communication for Reminder System, 2010, International Journal of Computer Application (09758887).

[9] Michael Batty, Andrew Hudson-Smith, Richard Milton and Andrew Crooks, "Map mashups, Web 2.0 and the GIS revolution," Annals of GIS. 2010.

[10] Amin Abdalla, "LatYourLife: A Geo-Temporal Task Planning Application,” 2012. 